\title{
A Remark on the Deformation of GNS Representations of *-Algebras
}

\author{
Stefan Waldmann* \\ Fakultät für Physik \\ Albert-Ludwigs-Universität Freiburg \\ Hermann Herder Straße 3 \\ D 79104 Freiburg \\ Germany
}

December 2000

FR-THEP 2000/18

\begin{abstract}
Motivated by deformation quantization we investigate the algebraic GNS construction of *representations of deformed *-algebras over ordered rings and compute their classical limit. The question if a GNS representation can be deformed leads to the deformation of positive linear functionals. Various physical examples from deformation quantization like the Bargmann-Fock and the Schrödinger representation as well as KMS functionals are discussed.
\end{abstract}

\section{Introduction}

Deformation quantization as introduced in [2] has shown to be not only a powerful quantization scheme of finite-dimensional systems, see [3, 4, 19, 22, 27, 30 34 for existence and classification results, but it also yields various applications to string theory and non-commutative field theories, see e.g. [16, 28, 29, 35], index theory [23, 32, 33], quantum field theory [18, 20, 21], and quantization of systems with constraints and phase space reduction [5, 24.

While the deformed algebras yield models for the observables, a notion of states by using positive linear functionals was introduced in [13] and used to obtain an algebraic version of the GNS construction of *-representations. In various examples this concept of *-representations on pre-Hilbert spaces over the formal power series was shown to be physically reasonable and mathematically fruitful [6] 8, 36].

In this article we continue the more systematic investigation of the ${ }^{*}$-representation theory of (deformed) *-algebras as started in [14 17], where the notion of a deformed ${ }^{*}$-representation was introduced. Here the positivity of the inner product of the pre-Hilbert space plays a crucial role. Since the GNS representations are certainly the most important ones we shall focus on this subclass of *-representations. To this end we study deformations of positive functionals and relate the GNS representations of the deformed and undeformed positive linear functionals: the classical limit of the GNS representation of the deformed functional gives back the GNS representation of the

\footnotetext{
*Stefan.Waldmann@physik.uni-freiburg.de
} 
undeformed one. This statement is in so far non-trivial as it involves a two-step quotient procedure where in the intermediate step the quotients are not the same. Here the notion of the classical limit of a pre-Hilbert space in the sense of [14. Sect. 8] comes in crucially. It also gives an easy criterion whether a GNS representation can be deformed: one only has to deform the corresponding positive linear functional which always can be done in the case of deformation quantization of symplectic manifolds [17, Prop. 5.1]. We explain and illustrate this in several examples like the BargmannFock representation and Schrödinger representation and point out the importance of the notion of a positive deformation of a *algebra.

The paper is organized as follows: In Section 2 we recall some basic facts on ${ }^{*}$-algebras and the GNS construction. Section 3 contains the definition and first properties of the deformation theory of ${ }^{*}$-algebras and their ${ }^{*}$-representations. In Section 4 we prove the main result and in Section 5 several examples are discussed.

\section{$2{ }^{*}$-Algebras and the GNS construction}

Let us briefly recall the notion of *-algebras over ordered rings and the GNS construction of * representations out of a positive linear functional, see 12 14 for more details.

By $\mathrm{R}$ we denote an ordered ring where our main examples needed for physical applications are $\mathbb{R}$ and $\mathbb{R}[[\lambda]]$. Then $C=R(i)$ with $i^{2}=-1$ will be the generalization of the complex numbers. If $R$ is ordered then $\mathrm{R}[[\lambda]]$ is ordered, too, whence this notion is well adapted to formal deformation theory. A pre-Hilbert space $\mathcal{H}$ over $\mathrm{C}$ is a $\mathrm{C}$-module with a positive definite Hermitian inner product, i.e. a map $\langle\cdot, \cdot\rangle: \mathcal{H} \times \mathcal{H} \rightarrow \mathrm{C}$ which is $\mathrm{C}$-linear in the second argument and satisfies $\langle\phi, \psi\rangle=\overline{\langle\psi, \phi\rangle}$ and $\langle\chi, \chi\rangle>0$ for all $\phi, \psi, \chi \in \mathcal{H}, \chi \neq 0$. Note that this notion is meaningful since $\mathrm{R}$ is ordered. By $\mathcal{B}(\mathcal{H})$ we denote the algebra of endomorphism of $\mathcal{H}$ having an adjoint with respect to $\langle\cdot, \cdot\rangle$. A * algebra $\mathcal{A}$ over $\mathrm{C}$ is an associative algebra over $\mathrm{C}$ with an involutive anti-linear anti-automorphism called the ${ }^{*}$-involution, which shall be denoted by $A \mapsto A^{*}$. In particular, $\mathcal{B}(\mathcal{H})$ is a ${ }^{*}$-algebra and $A^{*}$ is simply given by the adjoint of $A$. $\mathrm{A}^{*}$-representation of $\mathcal{A}$ on $\mathcal{H}$ is a ${ }^{*}$-homomorphism $\pi: \mathcal{A} \rightarrow \mathcal{B}(\mathcal{H})$.

A C-linear functional $\omega: \mathcal{A} \rightarrow \mathrm{C}$ is called positive if for all $A \in \mathcal{A}$

$$
\omega\left(A^{*} A\right) \geq 0 .
$$

In this case $\omega$ satisfies a Cauchy-Schwarz inequality, see e.g. [13, Lem. 5], and the space

$$
\mathcal{J}_{\omega}:=\left\{A \in \mathcal{A} \mid \omega\left(A^{*} A\right)=0\right\}
$$

turns out to be a left ideal in $\mathcal{A}$, the so-called Gel'fand ideal. Thus one has a $\mathcal{A}$-left module structure on $\mathcal{H}_{\omega}=\mathcal{A} / \mathcal{J}_{\omega}$ which is traditionally denoted by

$$
\pi_{\omega}(A) \psi_{B}=\psi_{A B},
$$

where $\psi_{B} \in \mathcal{H}$ denotes the equivalence class of $B \in \mathcal{A}$. Moreover, $\mathcal{H}_{\omega}$ is endowed with a pre-Hilbert space structure by setting

$$
\left\langle\psi_{A}, \psi_{B}\right\rangle=\omega\left(A^{*} B\right) .
$$

Then $\pi_{\omega}$ is easily shown to be a ${ }^{*}$-representation of $\mathcal{A}$ on $\mathcal{H}_{\omega}$, the so-called GNS representation corresponding to $\omega$. If $\mathcal{A}$ is unital then $\pi_{\omega}$ is cyclic with cyclic vector $\psi_{1}$ and we have $\omega(A)=$ $\left\langle\psi_{1}, \pi_{\omega}(A) \psi_{1}\right\rangle$, a property which characterizes $\pi_{\omega}$ up to unitary equivalence. Hence it will be convenient to consider unital *algebras in the following. See [14, 15] for results in the non-unital case and [6 $6,12,13,16,36]$ for various applications to deformation quantization. 


\section{Deformations of *-Algebras and *-Representations}

Now consider a formal associative deformation $\mathcal{A}=(\mathcal{A}[[\lambda]], \star)$ of a ${ }^{*}$-algebra $\mathcal{A}$ over $\mathrm{C}$ in the sense of Gerstenhaber [25, 26], i.e. $\star$ is an associative product deforming the product of $\mathcal{A}$ in higher orders of $\lambda$. Then this deformation is called Hermitian if the ${ }^{*}$-involution of $\mathcal{A}$ (extended $\mathrm{C}[[\lambda]]$-anti-linearly to $\mathcal{A}[[\lambda]])$ is still a ${ }^{*}$-involution for $\star$, see [17, Sect. 3]. In principle one can also deform the ${ }^{*}$-involution but in the physical applications this is not necessary and even not wanted: in deformation quantization $\mathcal{A}$ will play the role of the classical observable algebra and Hermitian elements in $\mathcal{A}$ correspond to measurable physical quantities, the observables. Thus this characterization should be preserved under quantization. Here the formal parameter $\lambda$ corresponds to Planck's constant $\hbar$ whenever the formal series converge.

Suppose now that $\mathcal{H}$ is a pre-Hilbert space over $C[[\lambda]]$. Then we consider the space $\mathcal{H}_{0}:=\{\phi \in$ $\left.\mathcal{H}|\langle\phi, \phi\rangle|_{\lambda=0}=0\right\}$. It turns out that $\mathcal{H}_{0}$ is a $\mathrm{C}[[\lambda]]$-submodule and $\mathcal{H}:=\mathfrak{C H}:=\mathfrak{H} / \mathcal{H}_{0}$, now viewed as a $\mathrm{C}$-module, is a pre-Hilbert space over $\mathrm{C}$. Indeed, if $\mathfrak{C}: \mathcal{H} \rightarrow \mathcal{H}$ denotes the projection then the inner product is just given by $\langle\mathfrak{C} \phi, \mathfrak{C} \psi\rangle=\left.\langle\phi, \boldsymbol{\psi}\rangle\right|_{\lambda=0}$, where $\phi, \boldsymbol{\psi} \in \mathcal{H}$. Finally, this procedure is functorial whence we shall refer to $\mathfrak{C}$ as the classical limit functor [14, Sect 8].

Example 3.1 Let $\mathcal{H}$ be a pre-Hilbert space over $\mathrm{C}$ and consider the $\mathrm{C}[[\lambda]]$-module $\mathcal{H}$. By $\lambda$-linear extension of the inner product, $\mathcal{H}$ becomes a pre-Hilbert space over $\mathrm{C}[[\lambda]]$. It follows immediately that $\mathcal{H}_{0}=\lambda \mathcal{H}=\lambda \mathcal{H}[[\lambda]]$ and thus $\mathfrak{C H} \cong \mathcal{H}$ again. Moreover, we note that in this case

$$
\mathcal{B}(\mathcal{H})=\mathcal{B}(\mathcal{H})[[\lambda]]
$$

However, the classical limit functor is defined on all pre-Hilbert spaces over $C[[\lambda]]$ and, in fact, we shall need the more general situation in the sequel.

If $\boldsymbol{\pi}$ is a ${ }^{*}$-representation of $\mathcal{A}$ on some pre-Hilbert space $\mathcal{H}$ over $\mathrm{C}[[\lambda]]$ then we can also define the classical limit $\pi=\mathfrak{C} \pi$ of the representation by setting $\pi(A) \mathfrak{C} \psi=\mathfrak{C}(\boldsymbol{\pi}(A) \boldsymbol{\psi})$. One obtains a *-representation $\pi$ of $\mathcal{A}$ on $\mathcal{H}=\mathfrak{C H}$. Again, $\mathfrak{C}$ is functorial, see [17, Sect 8].

This raises the following questions: For a given deformation $\mathcal{A}$ of $\mathcal{A}$, which ${ }^{*}$-representations of $\mathcal{A}$ occur as classical limits of ${ }^{*}$-representations of $\mathcal{A}$, i.e. which ${ }^{*}$-representations can be quantized? Moreover, one wants to understand in how many ways (up to unitary equivalence) this is possible and which properties are preserved under the classical limit and quantization.

A more restricted problem is the following: given a ${ }^{*}$-representation $(\mathcal{H}, \pi)$ of $\mathcal{A}$ can we find a ${ }^{*}$-representation $\boldsymbol{\pi}$ of $\mathcal{A}$ on $\mathcal{H}=\mathcal{H}[[\lambda]]$ with $\mathfrak{C} \boldsymbol{\pi}=\pi$ ? It turns out that this leads too quickly into obstructions and is thus too restrictive:

Consider the polynomials $\mathcal{A}=\mathbb{C}[z, \bar{z}]$ in two variables with the ${ }^{*}$-involution $z^{*}=\bar{z}$. Moreover, let $\mathcal{H}=\mathbb{C}$ with the standard inner product and $\pi(1)=\mathrm{id}, \pi(z)=0=\pi(\bar{z})$. This determines a *-representation of $\mathcal{A}$ on $\mathcal{H}$. Now consider the Wick product

$$
f *_{\text {Wick }} g=\sum_{r=0}^{\infty} \frac{(2 \lambda)^{r}}{r !} \frac{\partial^{r} f}{\partial z^{r}} \frac{\partial^{r} g}{\partial \bar{z}^{r}}
$$

for $f, g \in \mathcal{A}[[\lambda]]$ which gives a Hermitian deformation, see e.g. [11]. Then a simple and straightforward computation shows the following lemma:

Lemma 3.2 There is $n o{ }^{*}$-representation $\boldsymbol{\pi}$ of $\left(\mathcal{A}[[\lambda]], *_{\text {Wick }}\right)$ on $\mathbb{C}[[\lambda]]$ deforming $\pi$.

Proof: Suppose there would be such a $\boldsymbol{\pi}$. Then necessarily $\boldsymbol{\pi}(1)=$ id and $\boldsymbol{\pi}(z)$ as well as $\boldsymbol{\pi}(\bar{z})$ would have to start in order $\lambda$. But this is incompatible with $z *_{\text {wick }} \bar{z}-\bar{z} *_{\text {Wick }} z=2 \lambda 1$. 


\section{Deformation of GNS representations}

Since the problem of deforming a ${ }^{*}$-representation $(\mathcal{H}, \pi)$ of $\mathcal{A}$ in full generality seems to be rather difficult we shall now restrict to a more specific class of representations, namely the GNS representations.

Hence let $\omega: \mathcal{A} \rightarrow \mathrm{C}$ be a positive linear functional and let $\mathcal{A}=(\mathcal{A}[[\lambda]], \star)$ be a Hermitian deformation of $\mathcal{A}$. Moreover, let $\boldsymbol{\omega}: \mathcal{A} \rightarrow \mathrm{C}[[\lambda]]$ be a $\mathrm{C}[[\lambda]]$-linear functional which therefore can be written as $\boldsymbol{\omega}=\sum_{r=0}^{\infty} \lambda^{r} \boldsymbol{\omega}_{r}$ with $\boldsymbol{\omega}_{r}: \mathcal{A} \rightarrow$ C. It is called a deformation of $\boldsymbol{\omega}$ if $\boldsymbol{\omega}$ is a positive functional for $\mathcal{A}$ and $\boldsymbol{\omega}_{0}=\omega$. In this situation we can compute the classical limit of the GNS representation induced by $\boldsymbol{\omega}$ :

Theorem 4.1 Let $\mathcal{A}$ be $a^{*}$-algebra over $\mathrm{C}$ and $\mathcal{A}=(\mathcal{A}[[\lambda]], \star)$ a Hermitian deformation of $\mathcal{A}$. If $\boldsymbol{\omega}: \mathcal{A} \rightarrow \mathrm{C}[[\lambda]]$ is a deformation of a positive linear functional $\omega=\boldsymbol{\omega}_{0}: \mathcal{A} \rightarrow \mathrm{C}$ then the classical limit of the GNS representation $\left(\mathcal{H}_{\boldsymbol{\omega}}, \boldsymbol{\pi}_{\boldsymbol{\omega}}\right)$ is canonically unitarily equivalent to the GNS representation $\left(\mathcal{H}_{\omega}, \pi_{\omega}\right)$ by the intertwiner

$$
U: \mathfrak{C H}_{\boldsymbol{\omega}} \ni \mathfrak{C} \psi_{\boldsymbol{A}} \mapsto \psi_{A} \in \mathcal{H}_{\omega}
$$

where $\boldsymbol{A}=\sum_{r=0}^{\infty} \lambda^{r} \boldsymbol{A}_{r} \in \mathcal{A}$ with $A=\boldsymbol{A}_{0}$.

Proof: It is straightforward to check that $U$ is well-defined, unitary, and an intertwiner between $\mathfrak{C} \pi_{\boldsymbol{\omega}}$ and $\pi_{\omega}$.

This result is in so far non-trivial as the relation between the two Gel'fand ideals $\mathcal{J}_{\boldsymbol{\omega}}$ and $\mathcal{J}_{\omega}$ may be quite complicated. Typically, there is no isomorphism like $\mathcal{J}_{\boldsymbol{\omega}} \cong \mathcal{J}_{\omega}[[\lambda]]$ inside $\mathcal{A}[[\lambda]]$ : Using the terminology of [5. Def. 30] the Gel'fand ideal $\mathcal{J}_{\omega}$ is not a deformation of the submodule $\mathcal{J}_{\omega}$ in general.

This can be understood heuristically as follows. From $\boldsymbol{\omega}\left(A^{*} \star A\right)=0$ one obtains usually more conditions than only the zeroth order condition $\omega\left(A^{*} A\right)=0$. Hence $\mathfrak{J}_{\boldsymbol{\omega}}$ is typically 'smaller' than $\mathcal{J}_{\omega}[[\lambda]]$. Thus the pre-Hilbert space $\mathcal{H}_{\omega}$ is 'bigger' than $\mathcal{H}_{\omega}[[\lambda]]$. However, the 'additional' vectors in $\mathcal{H}_{\boldsymbol{\omega}}$ have inner products which vanish in the classical limit $\lambda=0$. Thus $\mathfrak{C F}_{\boldsymbol{\omega}} \cong \mathcal{H}_{\boldsymbol{\omega}}$ becomes possible. Note however, that in general there is no canonical way to compare $\mathcal{H}_{\boldsymbol{\omega}}$ with $\mathcal{H}_{\omega}[[\lambda]]$.

According to the above theorem the problem of deforming a representation reduces to the deformation of a positive functional, at least in the case of a GNS representation. Hence we shall investigate which classically positive functionals can actually be deformed.

\section{$5 \quad$ Examples}

A first example of a deformed GNS representation is given by faithful positive linear functionals. A positive linear functional $\omega: \mathcal{A} \rightarrow \mathrm{C}$ is called faithful if $\omega\left(A^{*} A\right)>0$ for all $A \neq 0$. It follows $\partial_{\omega}=\{0\}$ and hence $\mathcal{H}_{\omega}=\mathcal{A}$ as C-modules. The GNS representation $\pi_{\omega}$ is just the usual left-action. Then the following statement is obvious:

Lemma 5.1 Let $\omega: \mathcal{A} \rightarrow C$ be a faithful positive linear functional and let $\boldsymbol{\omega}=\sum_{r=0}^{\infty} \lambda^{r} \boldsymbol{\omega}_{r}: \mathcal{A} \rightarrow$ $\mathrm{C}[[\lambda]]$ be a real $\mathrm{C}[[\lambda]]$-linear functional (i.e. $\boldsymbol{\omega}\left(A^{*}\right)=\overline{\boldsymbol{\omega}(A)}$ ) with $\boldsymbol{\omega}_{0}=\omega$. Then $\boldsymbol{\omega}$ is a faithful positive $\mathrm{C}[[\lambda]]$-linear functional of $\mathcal{A}$ and thus a deformation of $\omega$.

Thus a faithful positive linear functional can always be deformed whence the above theorem can be applied. 
The physical importance of faithful positive functionals comes from the fact that in deformation quantization these functionals correspond to thermodynamical states. In particular the $K M S$ functionals on symplectic manifolds are faithful, see [1, 9, 10, 36] for further details.

On the other hand there are strong obstructions on the ${ }^{*}$-algebra $\mathcal{A}$ to have a faithful positive linear functional at all: in particular, it follows immediately that the minimal ideal $\mathcal{J}_{\text {min }}(\mathcal{A})$, which is the intersection of all Gel'fand ideals, has to be trivial. For a detailed discussion and further consequences of this matter we refer to 15 .

Another important case is obtained if the deformation $\mathcal{A}$ is a positive deformation of $\mathcal{A}$, i.e. if any positive linear functional $\omega: \mathcal{A} \rightarrow C$ can be deformed [17, Def. 4.1]. In this case we have the following result:

Proposition 5.2 Let $\mathcal{A}$ be a positive deformation of a unital ${ }^{*}$-algebra $\mathcal{A}$ over $\mathrm{C}$. If $(\mathcal{H}, \pi)$ is an orthogonal sum of cyclic (and hence $G N S){ }^{*}$-representations then $(\mathcal{H}, \pi)$ can be deformed into a ${ }^{*}$-representation for $\mathcal{A}$.

Proof: This follows immediately from the fact that the classical limit functor preserves orthogonal sums.

Unfortunately, in general there may be ${ }^{*}$-representations of $\mathcal{A}$ which are not orthogonal sums of cyclic ones. Even for $C^{*}$-algebras one needs to complete a direct sum in order to obtain all (non-degenerate) representations. However, in physical applications of deformation quantization, where $\mathcal{A}$ plays the role of the observable algebra, this can typically be assumed in order to obtain interesting representations. Here a cyclic vector has the interpretation of the 'vacuum vector' or 'ground state': It should be possible to obtain all vectors by applying the observables to the ground state. Furthermore, it was shown in [17, Prop. 5.1] that star products on symplectic manifolds are automatically positive deformations. Thus the above situation applies to these star products of deformation quantization.

Let us now discuss more concrete examples from deformation quantization. First we consider the Wick star product $*_{\text {wick }}$ on $\mathbb{C}^{n}$ which generalizes (3.2) to $C^{\infty}\left(\mathbb{C}^{n}\right)[[\lambda]]$, see e.g. [11]. It is known that $*_{\text {Wick }}$ is even a strongly positive deformation, i.e. all classically positive linear functionals are positive for $*_{\text {Wick }}$ as well [17, Lem. 4.4]. In particular, the $\delta$-functional at $0 \in \mathbb{C}^{n}$ is positive and yields the Bargmann-Fock representation [13. We shall discuss this example now in detail to illustrate the above introduced notions. For the classical and quantum Gel'fand ideals $\mathcal{J}$ and $\mathcal{J}$ one obtains

$$
\mathcal{J}=\left\{f \in C^{\infty}\left(\mathbb{C}^{n}\right) \mid f(0)=0\right\} \quad \text { and } \quad \mathcal{J}=\left\{f \in C^{\infty}\left(\mathbb{C}^{n}\right)[[\lambda]] \mid \forall I \ni \mathbb{N}^{n}: \frac{\partial^{|I|} f}{\partial \bar{z}^{I}}(0)=0\right\}
$$

respectively. Hence the representation spaces $\mathcal{H}$ and $\mathcal{H}$ are given by

$$
\mathcal{H}=\mathbb{C} \quad \text { and } \quad \mathcal{H}=\left(\mathbb{C}\left[\left[\bar{y}^{1}, \ldots, \bar{y}^{n}\right]\right]\right)[[\lambda]]
$$

with the usual $\mathbb{C}$-valued inner product for $\mathcal{H}$ and

$$
\langle\psi, \phi\rangle=\sum_{r=0}^{\infty} \frac{(2 \lambda)^{r}}{r !} \sum_{|I|=r} \overline{\frac{\partial^{r} \psi}{\partial \bar{y}^{I}}(0)} \frac{\partial^{r} \phi}{\partial \bar{y}^{I}}(0),
$$

for $\psi, \phi \in \mathcal{H}$. The GNS representation is classically given by $\pi(f) 1=f(0) 1$, analogously to Lemma 3.2. Quantum mechanically it is the usual Bargmann-Fock representation, in particular $\boldsymbol{\pi}\left(z^{k}\right)=2 \lambda \frac{\partial}{\partial \bar{y}^{k}}$ and $\boldsymbol{\pi}\left(\bar{z}^{k}\right)=\bar{y}^{k}$, see [13, Lem. 8]. Now we see that $\mathcal{J}$ is strictly smaller than $\mathcal{J}[[\lambda]]$ and $\mathcal{H}_{0}$ contains all vectors in $\mathcal{H}$ except the $\mathbb{C}$-multiples of 1 . Hence it is not just $\lambda \mathcal{H}$. Thus we see that 
with the more general notion of a deformation of a ${ }^{*}$-representation we can avoid the obstructions from Lemma 3.2. Let us also remark that the same phenomena occurs for the Wick star product on an arbitrary Kähler manifold $M$ and the $\delta$-functional at some $p \in M$, cf. [13]. We also remark that in this example the classical limit $\pi$ is 'more degenerate' than the quantized representation $\boldsymbol{\pi}$. On e.g. the polynomials on $\mathbb{C}^{n}$ the representation $\boldsymbol{\pi}$ is faithful while $\pi$ is not. See [14, Prop. 8.5] for a more detailed discussion of which properties are preserved under the classical limit.

In a last example we shall discuss the formal Schrödinger representation as discussed in [6, 8, 13]. We consider the cotangent bundle $\pi: T^{*} \mathbb{R}^{n} \rightarrow \mathbb{R}^{n}$ with the usual Weyl-Moyal star product

$$
f *_{\text {Weyl }} g=\mu \circ \mathrm{e}^{\frac{\mathrm{i} \lambda}{2} \sum_{k}\left(\frac{\partial}{\partial q^{k}} \otimes \frac{\partial}{\partial p_{k}}-\frac{\partial}{\partial p_{k}} \otimes \frac{\partial}{\partial q^{k}}\right)} f \otimes g,
$$

where $\mu(f \otimes g)=f g$ is the pointwise product of $f, g \in C^{\infty}\left(T^{*} \mathbb{R}^{n}\right)[[\lambda]]$. For convenience we consider compactly supported functions $f \in C_{0}^{\infty}\left(T^{*} \mathbb{R}^{n}\right)[[\lambda]]$ and use the Schrödinger functional

$$
\omega(f)=\int_{\mathbb{R}^{n}} \iota^{*} f d^{n} q,
$$

where $\iota: \mathbb{R}^{n} \hookrightarrow T^{*} \mathbb{R}^{n}$ is the zero section and $d^{n} q$ the usual volume form. Clearly $\omega$ is a classically positive linear functional and it turns out that $\omega$ is positive for $*_{\text {weyl }}$, too, without the need of further deformations. The Gel'fand ideals are given by

$$
\mathcal{J}=\left\{f \in C_{0}^{\infty}\left(T^{*} \mathbb{R}^{n}\right) \mid \iota^{*} f=0\right\} \quad \text { and } \quad \mathcal{J}=\left\{f \in C_{0}^{\infty}\left(T^{*} \mathbb{R}^{n}\right)[[\lambda]] \mid \iota^{*} N f=0\right\},
$$

respectively. Here $N=\exp \left(\frac{\lambda}{2 \mathrm{i}} \Delta\right)$ with $\Delta=\frac{\partial^{2}}{\partial q^{k} \partial p_{k}}$. Since $N$ is a bijection it follows that in this example $\mathcal{J}$ is a deformation of $\mathcal{J}$ as a subspace in the sense of [5. Def. 30]. Moreover, the representation spaces are just

$$
\mathcal{H}=C_{0}^{\infty}\left(\mathbb{R}^{n}\right) \quad \text { and } \quad \mathcal{H}=\mathcal{H}[[\lambda]]=C_{0}^{\infty}\left(\mathbb{R}^{n}\right)[[\lambda]]
$$

with the usual $L^{2}$ inner product induced by $d^{n} q$. Thus in this case we simply have $\mathcal{H}_{0}=\lambda \mathcal{H}$. Finally the ${ }^{*}$-representations are now given by

$$
\varrho(f) \psi=\iota^{*}\left(f \pi^{*} \psi\right)=\left(\iota^{*} f\right) \psi \quad \text { and } \quad \varrho(f) \psi=\iota^{*} N\left(f *_{\text {Weyl }} \pi^{*} \psi\right) .
$$

Again, these considerations are easily transfered to the general case of a cotangent bundle with the star product $*_{\text {Weyl }}$ as constructed in [6] 8$]$ and the results are literally the same. We shall omit the rather obvious details.

We conclude that in the case of the Schrödinger functional the correct classical limit can be obtained by naively 'setting $\lambda=0$ ' everywhere. On the other hand, in the Wick case this does not yield an acceptable result since then the classical representation space would be $\mathbb{C}\left[\left[\bar{y}^{1}, \ldots, \bar{y}^{n}\right]\right]$ with a highly degenerate inner product as obtained from (5.3) by 'setting $\lambda=0$ '. Thus one needs the more general construction of dividing by $\mathcal{H}_{0}$ in this case.

Physically speaking this can be interpreted as follows. The naive classical limit by setting $\lambda=0$ does not give meaningful results for the 'wave functions' themselves, i.e. for the elements in the representation space $\mathcal{H}$, but only for the measurable quantities, i.e. the Hermitian inner products. Hence they should be used to control the classical limit.

\section{Acknowledgments}

First I would like to thank Henrique Bursztyn for various discussions concerning this topic as well as for many helpful comments on the manuscript. Moreover, I would like to thank Pierre Bieliavsky, Martin Bordemann, Michel Cahen, Simone Gutt, and Cornelius Paufler for valuable remarks. 


\section{References}

[1] Basart, H., Flato, M., Lichnerowicz, A., Sternheimer, D.: Deformation Theory applied to Quantization and Statistical Mechanics. Lett. Math. Phys. 8 (1984), 483-394.

[2] Bayen, F., Flato, M., Frønsdal, C., Lichnerowicz, A., Sternheimer, D.: Deformation Theory and Quantization. Ann. Phys. 111 (1978), 61-151.

[3] Bertelson, M., Bieliavsky, P., Gutt, S.: Parametrizing Equivalence Classes of Invariant Star Products. Lett. Math. Phys. 46 (1998), 339-345.

[4] Bertelson, M., Cahen, M., Gutt, S.: Equivalence of Star Products. Class. Quantum Grav. 14 (1997), A93-A107.

[5] Bordemann, M., Herbig, H.-C., Waldmann, S.: BRST Cohomology and Phase Space Reduction in Deformation Quantization. Commun. Math. Phys. 210 (2000), 107-144.

[6] Bordemann, M., Neumaier, N., Pflaum, M. J., Waldmann, S.: On representations of star product algebras over cotangent spaces on Hermitian line bundles. Preprint Freiburg FR-THEP-98/24 math.QA/9811055 (November 1998).

[7] Bordemann, M., Neumaier, N., Waldmann, S.: Homogeneous Fedosov Star Products on Cotangent Bundles I: Weyl and Standard Ordering with Differential Operator Representation. Commun. Math. Phys. 198 (1998), 363-396.

[8] Bordemann, M., Neumaier, N., Waldmann, S.: Homogeneous Fedosov star products on cotangent bundles II: GNS representations, the WKB expansion, traces, and applications. J. Geom. Phys. 29 (1999), 199-234.

[9] Bordemann, M., Römer, H., Waldmann, S.: A Remark on Formal KMS States in Deformation Quantization. Lett. Math. Phys. 45 (1998), 49-61.

[10] Bordemann, M., Römer, H., Waldmann, S.: KMS States and Star Product Quantization. Rep. Math. Phys. 44 (1999), 45-52.

[11] Bordemann, M., Waldmann, S.: A Fedosov Star Product of Wick Type for Kähler Manifolds. Lett. Math. Phys. 41 (1997), 243-253.

[12] Bordemann, M., Waldmann, S.: Formal GNS Construction and WKB Expansion in Deformation Quantization. In: Sternheimer, D., Rawnsley, J., Gutt, S. (eds.): Deformation Theory and Symplectic Geometry, Mathematical Physics Studies no. 20, 315-319. Kluwer Academic Publisher, Dordrecht, Boston, London, 1997.

[13] Bordemann, M., Waldmann, S.: Formal GNS Construction and States in Deformation Quantization. Commun. Math. Phys. 195 (1998), 549-583.

[14] Bursztyn, H., Waldmann, S.: Algebraic Rieffel Induction, Formal Morita Equivalence and Applications to Deformation Quantization. Preprint math.QA/9912182 (December 1999). To appear in J. Geom. Phys.

[15] Bursztyn, H., Waldmann, S.: *-Ideals and Formal Morita Equivalence of *-Algebras. Preprint math.QA/0005227 (May 2000). To appear in Int. J. Math.

[16] Bursztyn, H., Waldmann, S.: Deformation Quantization of Hermitian Vector Bundles. Preprint math.QA/0009170 (September 2000). To appear in Lett. Math. Phys.

[17] Bursztyn, H., Waldmann, S.: On Positive Deformations of ${ }^{*}$-Algebras. In: Dito, G., SternHeIMer, D. (EDS.): Confèrence Moshè Flato 1999. Quantization, Deformations, and Symmetries, Mathematical Physics Studies no. 22, 69-80. Kluwer Academic Publishers, Dordrecht, Boston, London, 2000 .

[18] Cattaneo, A., Felder, G.: A Path Integral Approach to the Kontsevich Quantization Formula. Commun. Math. Phys. 212 (2000), 591-611. 
[19] DeWilde, M., Lecomte, P. B. A.: Existence of Star-Products and of Formal Deformations of the Poisson Lie Algebra of Arbitrary Symplectic Manifolds. Lett. Math. Phys. 7 (1983), 487-496.

[20] Dito, J.: Star-Product Approach to Quantum Field Theory: The Free Scalar Field. Lett. Math. Phys. 20 (1990), 125-134.

[21] Dütsch, M., Fredenhagen, K.: Algebraic Quantum Field Theory, Perturbation Theory, and the Loop Expansion. Preprint hep-th/0001129 (January 2000).

[22] Fedosov, B. V.: A Simple Geometrical Construction of Deformation Quantization. J. Diff. Geom. 40 (1994), 213-238.

[23] Fedosov, B. V.: Deformation Quantization and Index Theory. Akademie Verlag, Berlin, 1996.

[24] Fedosov, B. V.: Non-Abelian Reduction in Deformation Quantization. Lett. Math. Phys. 43 (1998), $137-154$.

[25] Gerstenhaber, M.: On the Deformation of Rings and Algebras. Ann. Math. 79 (1964), 59-103.

[26] Gerstenhaber, M., Schack, S. D.: Algebraic Cohomology and Deformation Theory. In: Hazewinkel, M., Gerstenhaber, M. (eds.): Deformation Theory of Algebras and Structures and Applications, 13-264. Kluwer Academic Press, Dordrecht, 1988.

[27] Gutt, S., Rawnsley, J.: Equivalence of star products on a symplectic manifold; an introduction to Deligne's Čech cohomology classes. J. Geom. Phys. 29 (1999), 347-392.

[28] Jurco, B., Schupp, P.: Noncommutative Yang-Mills from equivalence of star products. Eur. Phys. J. C14 (2000), 367-370.

[29] Jurco, B., Schupp, P., Wess, J.: Noncommutative gauge theory for Poisson manifolds. Nucl. Phys. B584 (2000), 784-794.

[30] Kontsevich, M.: Deformation Quantization of Poisson Manifolds, I. Preprint q-alg/9709040 (September 1997).

[31] Kontsevich, M.: Operads and Motives in Deformation Quantization. Lett. Math. Phys. 48 (1999), $35-72$.

[32] Nest, R., Tsygan, B.: Algebraic Index Theorem. Commun. Math. Phys. 172 (1995), 223-262.

[33] Nest, R., Tsygan, B.: Algebraic Index Theorem for Families. Adv. Math. 113 (1995), 151-205.

[34] Omori, H., Maeda, Y., Yoshioka, A.: Weyl Manifolds and Deformation Quantization. Adv. Math. 85 (1991), 224-255.

[35] Schomerus, V.: D-branes and deformation quantization. JHEP 06 (1999).

[36] Waldmann, S.: Locality in GNS Representations of Deformation Quantization. Commun. Math. Phys. 210 (2000). 